\title{
Boundary value problems with eigenvalue-dependent boundary and transmission conditions
}

Kadriye Aydemir ${ }^{*}$

\author{
"Correspondence: \\ kadriye.aydemir@gop.edu.tr \\ Department of Mathematics, \\ Faculty of Arts and Science, \\ Gaziosmanpaşa University, Tokat, \\ 60250, Turkey
}

\begin{abstract}
In this paper the operator-theoretical method to investigate a new type boundary value problems consisting of a two-interval Sturm-Liouville equation together with boundary and transmission conditions dependent on eigenparameter is developed. By suggesting our own approach, we construct modified Hilbert spaces and a linear operator in them in such a way that the considered problem can be interpreted as a spectral problem for this operator. Then we introduce so-called left- and right-definite solutions and give a representation of solution of the corresponding nonhomogeneous problem in terms of these one-hand solutions. Finally, we construct Green's vector-function and investigate some important properties of the resolvent operator by using this Green's vector-function.
\end{abstract}

Keywords: Sturm-Liouville problems; eigenparameter-dependent boundary and transmission conditions; Green's function; resolvent operator

\section{Introduction}

Many important special equations which appear in physics, such as Airy's equation, Bessel's equation, wave equation, heat equation, Schrödinger's equation, Heun's equation, advection-dispersion equation, etc., are associated with Sturm-Liouville type operators. For instance, the one-dimensional form of the advection-dispersion equation for a nonreactive dissolved solute in a saturated, homogeneous, isotropic porous medium under steady, uniform flow is

$$
c_{t}+v c_{x}=D c_{x x}, \quad 0<x<L, t>0
$$

where $c(x, t)$ is the concentration of the solute, $v$ is the average linear groundwater velocity, $D$ is the coefficient of hydrodynamic dispersion, and $L$ is the length of the aquifer. Using the method of separation of variables, the problem can be written in the simplest SturmLiouville form

$$
\left[p(x) X^{\prime}\right]^{\prime}+\lambda r(x) X=0, \quad X(0)=0, \quad X^{\prime}(L)=0, \quad 0<x<L
$$

This example makes it clear that the Sturm-Liouville problems are of broad interest. There is a well-developed theory for classical Sturm-Liouville problems (see, e.g., [1-5] and the

\section{空 Springer}

○2014 Aydemir; licensee Springer. This is an Open Access article distributed under the terms of the Creative Commons Attribution License (http://creativecommons.org/licenses/by/2.0), which permits unrestricted use, distribution, and reproduction in any medium, provided the original work is properly cited. 
references therein). Details of the derivation of the theory and of related background results can be found in the cited references. Although the subject of Sturm-Liouville problems is over 160 years old, these problems are an intensely active field of research today. The main tool for solvability analysis of such problems is the concept of Green's function. Green's functions have played an important role as a theoretical tool in the field of physics, since the possibility of a transition from the problems in mathematical physics to integral equations is based on the fundamental concept of Green's function. Therefore, the powerful and unifying formalism of Green's functions finds applications not only in standard physics subjects such as perturbation and scattering theory, bound-state formation, etc., but also at the forefront of current and, most likely, future developments (see [6]). Green's function transforms the differential equation into the integral equation, which, at times, is more informative. In terms of Green's function, the BVP with arbitrary data can be solved in a form that shows clearly the dependence of the solution on the data. Namely, Green's function approach would allow us to have an integral representation of the solution instead of an infinite series. Determination of Green's functions is also possible using Sturm-Liouville theory. This leads to series representation of Green's functions (see, e.g., the monograph [1] as well as the recent results in [7] and the references therein).

Sturm-Liouville type problems with transmission conditions have become an important area of research in recent years because of the needs of modern technology, engineering and physics. Many of the mathematical problems encountered in the study of boundaryvalue-transmission problem cannot be treated with the usual techniques within the standard framework of boundary value problem (see [8-12]). In this study we shall consider a new type of Sturm-Liouville problems consisting of the two-interval Sturm-Liouville equation

$$
\mathcal{L}(u):=\left(-p(x) u^{\prime}(x)\right)^{\prime}+q(x) u(x)=\mu^{2} u(x), \quad x \in \Omega^{-} \cup \Omega^{+}
$$

together with eigenparameter-dependent boundary conditions of the form

$$
\begin{aligned}
& \ell_{1}(u):=\delta_{10} u(a)-\delta_{11} u^{\prime}(a)-\mu^{2}\left(\widetilde{\delta}_{10} u(a)-\widetilde{\delta}_{11} u^{\prime}(a)\right)=0, \\
& \ell_{2}(u):=\delta_{20} u(b)-\delta_{21} u^{\prime}(b)+\mu^{2}\left(\widetilde{\delta}_{20} u(b)-\widetilde{\delta}_{21} u^{\prime}(b)\right)=0,
\end{aligned}
$$

and eigenparameter-dependent transmission conditions at one interaction point $x=c$ of the form

$$
\begin{aligned}
\ell_{3}(u):= & \gamma_{10}^{+} u(c+)+\gamma_{10}^{-} u(c-)=0, \\
\ell_{4}(u):= & \gamma_{20}^{+} u(c+)+\gamma_{21}^{+} u^{\prime}(c+)+\gamma_{20}^{-} u(c-)+\gamma_{21}^{-} u^{\prime}(c-) \\
& -\mu^{2}\left(\tilde{\gamma}_{20}^{+} u(c+)+\widetilde{\gamma}_{21}^{+} u^{\prime}(c+)+\widetilde{\gamma}_{20}^{-} u(c-)+\widetilde{\gamma}_{21}^{-} u^{\prime}(c-)\right)=0,
\end{aligned}
$$

where $p(x)$ is a real-valued piecewise constant function, $p(x)=p^{-}>0$ for $x \in \Omega^{-}=[a, c)$, $p(x)=p^{+}>0$ for $x \in \Omega^{+}=(c, b]$, the potential $q(x)$ is a real-valued function continuous in each of the intervals $\Omega^{-}$and $\Omega^{+}$, and has finite limits $q(c \pm)=\lim _{x \rightarrow c \pm 0} q(x), \mu$ is a complex spectral parameter, the coefficients $\delta_{i j}, \widetilde{\delta}_{i j}, \gamma_{2 j}^{ \pm}, \widetilde{\gamma}_{2 j}^{ \pm}(i=1,2$ and $j=0,1), \gamma_{10}^{ \pm}$are real numbers. This Sturm-Liouville problem is a non-classical eigenvalue problem since the eigenvalue parameter $\mu$ appears not only in the differential equation, but also in the boundary and 
transmission conditions. Moreover, in the differential equation there appears a singularity at one interior point. Because of these reasons the spectral theory of this problem is more complicate. Naturally, eigenfunctions of this problem may have discontinuity at the singular interior point. Some special cases of this problem arise after an application of the Fourier method to a varied assortment of physical problems. For instance, some boundary value problems with transmission conditions arise in heat and mass transfer problems [13], in vibrating string problems when the string is loaded additionally with point masses [14], in diffraction problems [12], in quantum mechanics [15], in thermal conduction problems for a thin laminated plate [16] etc. Such properties as isomorphism, coerciveness with respect to the spectral parameter, completeness and Abel bases property of a system of root functions of some boundary value problems with transmission conditions and its applications to the corresponding initial boundary value problems for parabolic equations have been investigated in [16-19]. For the background and applications of boundary value transmission problems to different areas, we refer the reader to the monographs and some recent contribution $[8-11,17,18,20-25]$.

\section{Hilbert space formulation of the problem}

In certain cases the boundary value problem can be characterized by means of a uniquely determined unbounded self-adjoint operator. In these cases the eigenvalues and eigenfunctions of the boundary value problem are determined by the eigenvalues and eigenvectors of the corresponding operator; these will be called a self-adjoint case of the boundary value problem. In some cases such a characterization is not possible and these will be referred to as 'symmetric' cases in general. In classical point of view, our problem cannot be characterized as 'self-adjoint case'. For 'self-adjoint characterization' of the considered problem (1)-(5), we shall define a new Hilbert space as follows.

Denote the determinant of the $i$ th and $j$ th columns of the matrix

$$
\theta=\left[\begin{array}{cccc}
\gamma_{20}^{+} & \gamma_{21}^{+} & \gamma_{20}^{-} & \gamma_{21}^{-} \\
\tilde{\gamma}_{20}^{+} & \tilde{\gamma}_{21}^{+} & \tilde{\gamma}_{20}^{-} & \tilde{\gamma}_{21}^{-}
\end{array}\right]
$$

by $\left|\theta_{i j}\right|(1 \leq i<j \leq 4)$. Throughout the paper we shall assume that the conditions

$$
\begin{aligned}
& \gamma_{10}^{-}\left(\gamma_{10}^{-}\left|\theta_{12}\right|-\gamma_{10}^{+}\left|\theta_{23}\right|\right)>0, \quad \gamma_{10}^{+}\left(\gamma_{10}^{+}\left|\theta_{34}\right|-\gamma_{10}^{-}\left|\theta_{14}\right|\right)<0, \quad\left|\theta_{24}\right|=0, \\
& \rho_{1}=\operatorname{det}\left(\begin{array}{ll}
\delta_{11} & \delta_{10} \\
\widetilde{\delta}_{11} & \widetilde{\delta}_{10}
\end{array}\right)>0, \quad \rho_{2}=\operatorname{det}\left(\begin{array}{ll}
\delta_{21} & \delta_{20} \\
\widetilde{\delta}_{21} & \widetilde{\delta}_{20}
\end{array}\right)>0
\end{aligned}
$$

hold. Define a new inner-product space $\mathcal{H}$ as a direct sum space $\left(L_{2}\left(\Omega^{-}\right) \oplus L_{2}\left(\Omega^{+}\right)\right) \oplus C^{3}$ equipped with the modified inner-product

$$
\begin{aligned}
\langle U, V\rangle_{\mathcal{H}}:= & -\frac{1}{p^{-}}\left(\left|\theta_{34}\right|-\frac{\gamma_{10}^{-}}{\gamma_{10}^{+}}\left|\theta_{14}\right|\right) \int_{a}^{c-} u(x) \overline{v(x)} d x \\
& +\frac{1}{p^{+}}\left(\left|\theta_{12}\right|-\frac{\gamma_{10}^{+}}{\gamma_{10}^{-}}\left|\theta_{23}\right|\right) \int_{c+}^{b} u(x) \overline{v(x)} d x \\
& -\frac{1}{\rho_{1}}\left(\left|\theta_{34}\right|-\frac{\gamma_{10}^{-}}{\gamma_{10}^{+}}\left|\theta_{14}\right|\right) u_{1} \bar{v}_{1}+\frac{1}{\rho_{2}}\left(\left|\theta_{12}\right|-\frac{\gamma_{10}^{+}}{\gamma_{10}^{-}}\left|\theta_{23}\right|\right) u_{2} \bar{v}_{2}+u_{3} \bar{v}_{3}
\end{aligned}
$$


for $U=\left(u(x), u_{1}, u_{2}, u_{3}\right), V=\left(v(x), v_{1}, v_{2}, v_{3}\right) \in\left(L_{2}\left(\Omega^{-}\right) \oplus L_{2}\left(\Omega^{+}\right)\right) \oplus C^{3}$. It is easy to see that the relation (6) really defines a new inner product in the direct sum space $\left(L_{2}\left(\Omega^{-}\right) \oplus\right.$ $\left.L_{2}\left(\Omega^{+}\right)\right) \oplus C^{3}$.

\section{Lemma $1 \mathcal{H}$ is a Hilbert space.}

Proof Let $U_{n}=\left(u_{n}(x), u_{1 n}, u_{2 n}, u_{3 n}\right), n=1,2, \ldots$, be any Cauchy sequence in $\mathcal{H}$. Then by (6) the sequences $\left(u_{n}(x)\right)$ and $\left(u_{1 n}, u_{2 n}, u_{3 n}\right)$ will be Cauchy sequences in the Hilbert spaces $L_{2}\left(\Omega^{-}\right) \oplus L_{2}\left(\Omega^{+}\right)$and $C^{3}$, respectively. Therefore they are convergent. Let $u_{0}(x)$ and $\left(u_{10}, u_{20}, u_{30}\right)$ be limits of these sequences, respectively. Defining $U_{0}=\left(u_{0}(x), u_{10}, u_{20}, u_{30}\right)$ we have that $U_{0} \in \mathcal{H}$ and $U_{n} \rightarrow U_{0}$ in $\mathcal{H}$. The proof is complete.

Let us now define the boundary and transmission functionals $B_{a}(u):=\delta_{10} u(a)-\delta_{11} u^{\prime}(a)$, $\widetilde{B}_{a}(u):=\widetilde{\delta}_{10} u(a)-\widetilde{\delta}_{11} u^{\prime}(a), B_{b}(u):=\delta_{20} u(b)-\delta_{21} u^{\prime}(b), \widetilde{B}_{b}(u):=\widetilde{\delta}_{20} u(b)-\widetilde{\delta}_{21} u^{\prime}(b), T_{c}(u):=$ $T_{c}^{-}(u)-T_{c}^{+}(u), \widetilde{T}_{c}(u):=\widetilde{T}_{c}^{-}(u)-\widetilde{T}_{c}^{+}(u)$, where $T_{c}^{ \pm}(u)=\gamma_{20}^{ \pm} u(c \pm)+\gamma_{21}^{ \pm} u^{\prime}(c \pm), \widetilde{T}_{c}^{ \pm}(u)=$ $\tilde{\gamma}_{20}^{ \pm} u(c \pm)+\tilde{\gamma}_{21}^{ \pm} u^{\prime}(c \pm)$ and the linear operator $\Re: \mathcal{H} \rightarrow \mathcal{H}$ with the domain

$$
\begin{aligned}
\operatorname{dom}(\Re):= & \left\{U=\left(u(x), u_{1}, u_{2}, u_{3}\right): u(x), u^{\prime}(x) \in A C_{\mathrm{loc}}\left(\Omega^{-}\right) \oplus A C_{\mathrm{loc}}\left(\Omega^{+}\right),\right. \\
& \text {with a finite left- and right-hand limits } u(c \mp 0) \text { and } u^{\prime}(c \mp 0) ; \\
& \left.\mathcal{L}(u) \in L_{2}[a, b] ; \ell_{3}(u)=0 ; u_{1}=\widetilde{B}_{a}(u) ; u_{2}=-\widetilde{B}_{b}(u) ; u_{3}=\widetilde{T}_{c}(u)\right\}
\end{aligned}
$$

and action low

$$
\Re\left(u(x), u_{1}, u_{2}, u_{3}\right)=\left(\mathcal{L} f, B_{a}(u), B_{b}(u), T_{c}(u)\right) .
$$

Then problem (1)-(5) can be written in the operator equation form as $\Re U=\mu^{2} U, U \in$ $\operatorname{dom}(\Re)$ in the Hilbert space $\mathcal{H}$.

Theorem 1 The linear operator $\Re$ is symmetric in the Hilbert space $\mathcal{H}$.

Proof By applying the method of [22] it is not difficult to prove that the operator $\Re$ is densely defined in $\mathcal{H}$, i.e., $\overline{\operatorname{dom}(\Re)}=\mathcal{H}$. Now let $U=\left(u(x), \widetilde{B}_{a}(u),-\widetilde{B}_{b}(u), \widetilde{T}_{c}^{-}(u)-\right.$ $\left.\widetilde{T}_{c}^{+}(u)\right), V=\left(v(x), \widetilde{B}_{a}(v),-\widetilde{B}_{b}(v), \widetilde{T}_{c}^{-}(v)-\widetilde{T}_{c}^{+}(v)\right) \in \operatorname{dom}(\Re)$. By partial integration we have

$$
\begin{aligned}
& \langle\Re U, V\rangle_{\mathcal{H}}-\langle U, \Re V\rangle_{\mathcal{H}} \\
& =\left(\left|\theta_{34}\right|-\frac{\gamma_{10}^{-}}{\gamma_{10}^{+}}\left|\theta_{14}\right|\right) W(u, \bar{v} ; a) \\
& \quad-\left(\left|\theta_{34}\right|-\frac{\gamma_{10}^{-}}{\gamma_{10}^{+}}\left|\theta_{14}\right|\right) W(u, \bar{v} ; c-)+\left(\left|\theta_{12}\right|-\frac{\gamma_{10}^{+}}{\gamma_{10}^{-}}\left|\theta_{23}\right|\right) W(u, \bar{v} ; b) \\
& \quad-\left(\left|\theta_{12}\right|-\frac{\gamma_{10}^{+}}{\gamma_{10}^{-}}\left|\theta_{23}\right|\right) W(u, \bar{v} ; c+)-\frac{1}{\rho_{1}}\left(\left|\theta_{34}\right|-\frac{\gamma_{10}^{-}}{\gamma_{10}^{+}}\left|\theta_{14}\right|\right)\left(B_{a}(u) \overline{\widetilde{B}_{a}(v)}-\widetilde{B}_{a}(u) \overline{\left.B_{a}(v)\right)}\right. \\
& \quad+\frac{1}{\rho_{2}}\left(\left|\theta_{12}\right|-\frac{\gamma_{10}^{+}}{\gamma_{10}^{-}}\left|\theta_{23}\right|\right)\left(\widetilde{B}_{b}(u) \overline{B_{b}(v)}-B_{b}(u) \widetilde{B}_{b}(v)\right) \\
& \quad+\left(T_{c}(u) \widetilde{T}_{c}(\bar{v})-\widetilde{T}_{c}(u) T_{c}(\bar{v})\right),
\end{aligned}
$$


where, as usual, $W(u, \bar{v} ; x)$ denotes the Wronskians of the functions $u$ and $\bar{v}$. From the definitions of boundary functionals we get that

$$
\begin{aligned}
& B_{a}(u) \overline{\widetilde{B}_{a}(v)}-\widetilde{B}_{a}(u) \overline{B_{a}(v)}=\rho_{1} W(u, \bar{v} ; a), \\
& \widetilde{B}_{b}(u) \overline{B_{b}(v)}-B_{b}(u) \overline{\widetilde{B}_{b}(v)}=-\rho_{2} W(u, \bar{v} ; b) .
\end{aligned}
$$

Further, taking in view the definition of $\Re$ and initial conditions (14)-(19) we can derive that

$$
\begin{aligned}
T_{c}(u) \widetilde{T}_{c}(\bar{v})-\widetilde{T}_{c}(u) T_{c}(\bar{v})= & \left(\left|\theta_{12}\right|-\frac{\gamma_{10}^{+}}{\gamma_{10}^{-}}\left|\theta_{23}\right|\right) W(u, \bar{v} ; c+) \\
& +\left(\left|\theta_{34}\right|-\frac{\gamma_{10}^{-}}{\gamma_{10}^{+}}\left|\theta_{14}\right|\right) W(u, \bar{v} ; c-) .
\end{aligned}
$$

Finally, substituting (8), (9) and (10) in (7) we obtain that

$$
\langle\Re U, V\rangle_{\mathcal{H}}=\langle U, \Re V\rangle_{\mathcal{H}} \quad \text { for all } U, V \in \operatorname{dom}(\Re) .
$$

The proof is complete.

Theorem 2 The linear operator $\Re$ is self-adjoint in $\mathcal{H}$.

Proof Since $\mathfrak{R}$ is symmetric and densely defined on $\mathcal{H}$, it is sufficient to show that if

$$
\langle\Re U, V\rangle_{\mathcal{H}}=\langle U, W\rangle_{\mathcal{H}}
$$

for all $U=\left(u(x), \widetilde{B}_{a}(u),-\widetilde{B}_{b}(u), \widetilde{T}_{c}^{-}(u)-\widetilde{T}_{c}^{+}(u)\right) \in D(\Re)$, then $V \in D(\Re)$ and $\Re V=W$, where $V=\left(v(x), \hbar_{1}, \hbar_{2}, \hbar_{3}\right)$ and $W=\left(w(x), \kappa_{1}, \kappa_{2}, \kappa_{3}\right)$. Writing equality (11) for all $U \in\left(C_{0}^{\infty}\left(\Omega^{-}\right) \oplus\right.$ $\left.C_{0}^{\infty}\left(\Omega^{+}\right)\right) \oplus C^{3} \subset D(\Re)$ by standard Sturm-Liouville theory, we find that $\langle\ell u, v\rangle_{L_{2}}=\langle u, w\rangle_{L_{2}}$ and $w(x)=\mathcal{L} \nu$. Then from equality (11) it follows that

$$
\begin{aligned}
\langle\mathcal{L} u, v\rangle_{L_{2}}= & \langle u, \mathcal{L} v\rangle_{L_{2}}-\frac{1}{\rho_{1}}\left(\left|\theta_{34}\right|-\frac{\gamma_{10}^{-}}{\gamma_{10}^{+}}\left|\theta_{14}\right|\right)\left(\widetilde{B}_{a}(u) \bar{\kappa}_{1}-B_{a}(u) \bar{\hbar}_{1}\right) \\
& -\frac{1}{\rho_{2}}\left(\left|\theta_{12}\right|-\frac{\gamma_{10}^{+}}{\gamma_{10}^{-}}\left|\theta_{23}\right|\right)\left(\widetilde{B}_{b}(u) \bar{\kappa}_{2}+B_{b}(u) \bar{\hbar}_{2}\right) \\
& +\left[\left(\widetilde{T}_{c}^{-}(u)-\widetilde{T}_{c}^{+}(u)\right) \bar{\kappa}_{3}-\left(T_{c}^{-}(u)-T_{c}^{+}(u)\right) \bar{\hbar}_{3}\right] .
\end{aligned}
$$

On the other hand, by two partial integrations we get

$$
\begin{aligned}
\langle\mathcal{L} u, v\rangle_{L_{2}}= & \langle u, \mathcal{L} v\rangle_{L_{2}}+\left(\left|\theta_{34}\right|-\frac{\gamma_{10}^{-}}{\gamma_{10}^{+}}\left|\theta_{14}\right|\right) W(u, \bar{v} ; a) \\
& -\left(\left|\theta_{34}\right|-\frac{\gamma_{10}^{-}}{\gamma_{10}^{+}}\left|\theta_{14}\right|\right) W(u, \bar{v} ; c-)+\left(\left|\theta_{12}\right|-\frac{\gamma_{10}^{+}}{\gamma_{10}^{-}}\left|\theta_{23}\right|\right) W(u, \bar{v} ; b) \\
& -\left(\left|\theta_{12}\right|-\frac{\gamma_{10}^{+}}{\gamma_{10}^{-}}\left|\theta_{23}\right|\right) W(u, \bar{v} ; c+) .
\end{aligned}
$$


Thus,

$$
\begin{aligned}
& -\frac{1}{\rho_{1}}\left(\left|\theta_{34}\right|-\frac{\gamma_{10}^{-}}{\gamma_{10}^{+}}\left|\theta_{14}\right|\right)\left(\widetilde{B}_{a}(u) \bar{\kappa}_{1}-B_{a}(u) \bar{\hbar}_{1}\right) \\
& \quad-\frac{1}{\rho_{2}}\left(\left|\theta_{12}\right|-\frac{\gamma_{10}^{+}}{\gamma_{10}^{-}}\left|\theta_{23}\right|\right)\left(\widetilde{B}_{b}(u) \bar{\kappa}_{2}+B_{b}(u) \bar{\hbar}_{2}\right) \\
& \quad+\left[\left(\widetilde{T}_{c}^{-}(u)-\widetilde{T}_{c}^{+}(u)\right) \bar{\kappa}_{3}-\left(T_{c}^{-}(u)-T_{c}^{+}(u)\right) \bar{\hbar}_{3}\right] \\
& =\left(\left|\theta_{34}\right|-\frac{\gamma_{10}^{-}}{\gamma_{10}^{+}}\left|\theta_{14}\right|\right) W(u, \bar{v} ; a)-\left(\left|\theta_{34}\right|-\frac{\gamma_{10}^{-}}{\gamma_{10}^{+}}\left|\theta_{14}\right|\right) W(u, \bar{v} ; c-) \\
& \quad+\left(\left|\theta_{12}\right|-\frac{\gamma_{10}^{+}}{\gamma_{10}^{-}}\left|\theta_{23}\right|\right) W(u, \bar{v} ; b)-\left(\left|\theta_{12}\right|-\frac{\gamma_{10}^{+}}{\gamma_{10}^{-}}\left|\theta_{23}\right|\right) W(u, \bar{v} ; c+) .
\end{aligned}
$$

From this equality, by applying the technique of Theorem 2.5 in our previous work [11], it can be derived easily that $\hbar_{1}=\widetilde{B}_{a}(v), \hbar_{2}=-\widetilde{B}_{b}(v), \hbar_{3}=\widetilde{T}_{c}^{-}(v)-\widetilde{T}_{c}^{+}(v), \ell_{3} v=0$ and $\kappa_{1}=B_{a}(w)$, $\kappa_{2}=B_{b}(w), \kappa_{3}=T_{c}^{-}(w)-T_{c}^{+}(w)$. The proof is complete.

Theorem 3 The operator $\Re$ has only point spectrum, i.e., $\sigma(\Re)=\sigma_{p}(\Re)$.

Proof It suffices to prove that if $\mu^{2}$ is not an eigenvalue of $\Re$, then $\mu^{2}$ is a regular point of $\Re$, i.e., $\mu^{2} \in \sigma(\Re)$. Let $\mu^{2}$ not be an eigenvalue of $\Re$. The resolvent operator $R\left(\mu^{2}, \Re\right)=$ $\left(\mu^{2} I-\Re\right)^{-1}$ exists and is defined on all of $\mathcal{H}$. By Theorem 2 and the closed graph theorem, we get that $R\left(\mu^{2}, \Re\right)$ is bounded. Thus, $\mu^{2} \in \sigma(\Re)$. Hence $\sigma(\Re)=\sigma_{p}(\Re)$.

\section{Left-definite and right-definite solutions}

In this section we shall define two basic solutions $v^{-}(x, \mu)$ and $\omega^{-}(x, \mu)$ on the left interval $\Omega^{-}$(so-called left-definite solutions) and two basic solutions $v^{+}(x, \mu)$ and $\omega^{+}(x, \mu)$ on the right interval $\Omega^{+}$(so-called right-definite solutions) by a special procedure as follows. Let $v^{-}(x, \mu)$ and $\omega^{+}(x, \mu)$ be solutions of equation (1) on $\Omega^{-}$and $\Omega^{+}$satisfying the initial conditions

$$
v^{-}(a, \mu)=\delta_{11}-\mu^{2} \widetilde{\delta}_{11}, \quad \frac{\partial v^{-}(a, \mu)}{\partial x}=\delta_{10}-\mu^{2} \widetilde{\delta}_{10}
$$

and

$$
\omega^{+}(b, \mu)=\delta_{21}+\mu^{2} \widetilde{\delta}_{21}, \quad \frac{\partial \omega^{+}(b, \mu)}{\partial x}=\delta_{20}+\mu^{2} \widetilde{\delta}_{20},
$$

respectively. By using these solutions we shall define the other solutions $v^{+}(x, \mu)$ and $\omega^{-}(x, \mu)$ by the initial conditions

$$
\begin{aligned}
v^{+}(c+, \mu)=- & \frac{\gamma_{10}^{-}}{\gamma_{10}^{+}} v^{-}(c-, \mu), \\
\frac{\partial v^{+}(c+, \mu)}{\partial x}= & \frac{1}{\gamma_{10}^{+}\left(\gamma_{21}^{+}-\mu^{2} \widetilde{\gamma}_{21}^{+}\right)}\left[\gamma_{10}^{+}\left(\mu^{2} \widetilde{\gamma}_{21}^{-}-\gamma_{21}^{-}\right) \frac{\partial v^{-}(c-, \mu)}{\partial x}\right. \\
& \left.+\left(\gamma_{10}^{+}\left(\mu^{2} \widetilde{\gamma}_{20}^{-}-\gamma_{20}^{-}\right)-\gamma_{10}^{-}\left(\mu^{2} \widetilde{\gamma}_{20}^{+}-\gamma_{20}^{+}\right)\right) v^{-}(c-, \mu)\right]
\end{aligned}
$$


and

$$
\begin{aligned}
\omega^{-}(c-, \mu)=- & \frac{\gamma_{10}^{+}}{\gamma_{10}^{-}} \omega^{+}(c+, \mu), \\
\frac{\partial \omega^{-}(c-, \mu)}{\partial x}= & \frac{1}{\gamma_{10}^{-}\left(\gamma_{21}^{-}-\mu^{2} \tilde{\gamma}_{21}^{-}\right)}\left[\gamma_{10}^{-}\left(\mu^{2} \widetilde{\gamma}_{21}^{+}-\gamma_{21}^{+}\right) \frac{\partial \omega^{+}(c+, \mu)}{\partial x}\right. \\
& \left.+\left(\gamma_{10}^{-}\left(\mu^{2} \tilde{\gamma}_{20}^{+}-\gamma_{20}^{+}\right)-\gamma_{10}^{+}\left(\mu^{2} \widetilde{\gamma}_{20}^{-}-\gamma_{20}^{-}\right)\right) \omega^{+}(c+, \mu)\right],
\end{aligned}
$$

respectively. The existence of these solutions follows from the well-known Cauchy-Picard theorem of ordinary differential equation theory. Moreover, by applying the method of [20], we can prove that each of these solutions are entire functions of the parameter $\mu \in C$ for each fixed $x$.

\section{Construction of Green's function}

In this section we develop the idea of a resolvent operator to solve nonhomogeneous boundary-value transmission problems (BVTP) as follows. Consider the operator equation

$$
\left(\mu^{2} I-\mathfrak{R}\right) U=F
$$

for arbitrary $F=\left(f(x), f_{1}, f_{2}, f_{3}\right) \in \mathcal{H}$. This operator equation is equivalent to the following nonhomogeneous BVTP:

$$
\begin{aligned}
& \left(\mu^{2}-\mathcal{L}\right) u(x)=f(x), \quad x \in \Omega^{-} \cup \Omega^{+}, \\
& \ell_{1}(u)=f_{1}, \quad \ell_{2}(u)=f_{2}, \quad \ell_{3}(u)=0, \quad \ell_{4}(u)=f_{3} .
\end{aligned}
$$

Let us define the Wronskians $\Delta^{-}(\mu):=W\left[v^{-}, \omega^{-}\right]_{x}$ for $x \in \Omega^{-}$and $\Delta^{+}(\mu):=W\left[v^{+}, \omega^{+}\right]_{x}$ for $x \in \Omega^{+}$and suppose that $\left(\gamma_{21}^{ \pm}-\mu^{2} \tilde{\gamma}_{21}^{ \pm}\right) \Delta^{ \pm}(\mu) \neq 0$. We shall search the resolvent function of this BVTP in the form

$$
u(x, \mu)= \begin{cases}\hbar_{11}(x, \mu) v^{-}(x, \mu)+\hbar_{12}(x, \mu) \omega^{-}(x, \mu) & \text { for } x \in \Omega^{-} \\ \hbar_{21}(x, \mu) v^{+}(x, \mu)+\hbar_{22}(x, \mu) \omega^{+}(x, \mu) & \text { for } x \in \Omega^{+}\end{cases}
$$

where the functions $\hbar_{11}(x, \mu)$ and $\hbar_{12}(x, \mu)$ are the solutions of the system of equations

$$
\left\{\begin{array}{l}
\frac{\partial \hbar_{11}(x, \mu)}{\partial x} v^{-}(x, \mu)+\frac{\partial \hbar_{12}(x, \mu)}{\partial x} \omega^{-}(x, \mu)=0 \\
\frac{\partial \hbar_{11}(x, \mu)}{\partial x} \frac{\partial v^{-}(x, \mu)}{\partial x}+\frac{\partial \hbar_{12}(x, \mu)}{\partial x} \frac{\partial \omega^{-}(x, \mu)}{\partial x}=\frac{f(x)}{p^{-}}
\end{array}\right.
$$

and the functions $\hbar_{21}(x, \mu), \hbar_{22}(x, \mu)$ are the solutions of the system of equations

$$
\left\{\begin{array}{l}
\frac{\partial \hbar_{21}(x, \mu)}{\partial x} v^{+}(x, \mu)+\frac{\partial d \hbar_{22}(x, \mu)}{\partial x} \omega^{+}(x, \mu)=0 \\
\frac{\partial \hbar_{21}(x, \mu)}{\partial x} \frac{\partial v^{+}(x, \mu)}{\partial x}+\frac{\partial \hbar_{22}(x, \mu)}{\partial x} \frac{\partial \omega^{+}(x, \mu)}{\partial x}=\frac{f(x)}{p^{+}}
\end{array}\right.
$$

for $x \in \Omega^{-}$and $x \in \Omega^{+}$, respectively. Since $\Delta^{-}(\mu) \neq 0$ and $\Delta^{+}(\mu) \neq 0$, from (24) and (25) we have

$$
\hbar_{11}(x, \mu)=\frac{1}{p^{-} \Delta^{-}(\mu)} \int_{x}^{c-} f(s) \omega^{-}(s, \mu) d s+\widetilde{\hbar}_{11}(\mu), \quad x \in \Omega^{-},
$$




$$
\begin{array}{ll}
\hbar_{12}(x, \mu)=\frac{1}{p^{-} \Delta^{-}(\mu)} \int_{a}^{x} f(s) v^{-}(s, \mu) d s+\widetilde{\hbar}_{12}(\mu), & x \in \Omega^{-}, \\
\hbar_{21}(x, \mu)=\frac{1}{p^{+} \Delta^{+}(\mu)} \int_{x}^{b} f(s) \omega^{+}(s, \mu) d s+\widetilde{\hbar}_{21}(\mu), & x \in \Omega^{+}, \\
\hbar_{22}(x, \mu)=\frac{1}{p^{+} \Delta^{+}(\mu)} \int_{c+}^{x} f(s) v^{+}(s, \mu) d s+\widetilde{\hbar}_{22}(\mu), & x \in \Omega^{+},
\end{array}
$$

where $\widetilde{\hbar}_{i j}(\mu)(i, j=1,2)$ are unknown functions depending only on the parameter $\mu$. Substituting into $(23)$ gives

$$
u(x, \mu)=\left\{\begin{array}{cc}
\frac{\omega^{-}(x, \mu)}{p^{-} \Delta^{-}(\mu)} \int_{a}^{x} v^{-}(s, \mu) f(s) d s+\frac{v^{-}(x, \mu)}{p^{-} \Delta^{-}(\mu)} \int_{x}^{c-} \omega^{-}(s, \mu) f(s) d s & \\
\quad+\widetilde{\hbar}_{11}(\mu) v^{-}(x, \mu)+\widetilde{\hbar}_{12}(\mu) \omega^{-}(x, \mu) & \text { for } x \in \Omega^{-}, \\
\frac{\omega^{+}(x, \mu)}{p^{+} \Delta^{+}(\mu)} \int_{c+}^{x} v^{+}(s, \mu) f(s) d s+\frac{v^{+}(x, \mu)}{p^{+} \Delta^{+}(\mu)} \int_{x}^{b} \omega^{+}(s, \mu) f(s) d s & \\
\quad+\widetilde{\hbar}_{21}(\mu) v^{+}(x, \mu)+\widetilde{\hbar}_{22}(\mu) \omega^{+}(x, \mu) & \text { for } x \in \Omega^{+} .
\end{array}\right.
$$

By differentiating we have

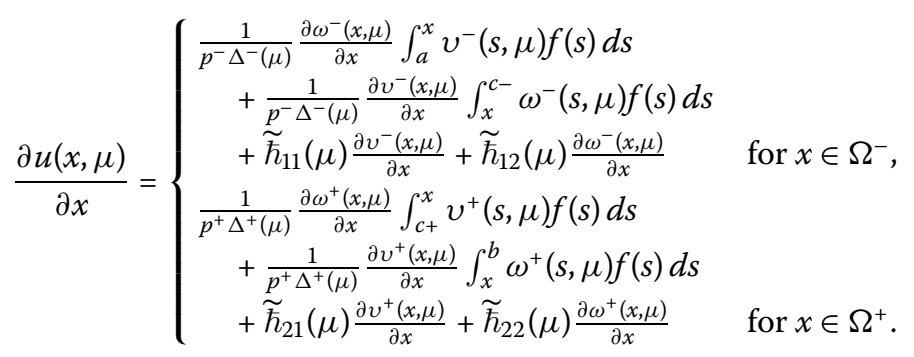

By using (26), (27) and conditions (22) we can derive that

$$
\begin{aligned}
& \widetilde{\hbar}_{11}(\mu)=\frac{1}{p^{+} \Delta^{+}(\mu)} \int_{c+}^{b} \omega^{+}(s, \mu) f(s) d s+\frac{f_{2}}{\Delta^{+}(\mu)}+\frac{f_{3}}{\gamma_{10}^{+}\left(\gamma_{21}^{+}-\mu^{2} \widetilde{\gamma}_{21}^{+}\right) \Delta^{+}(\mu)}, \\
& \tilde{\hbar}_{12}(\mu)=\frac{f_{1}}{\Delta^{-}(\mu)}, \quad \widetilde{\hbar}_{21}(\mu)=\frac{f_{2}}{\Delta^{+}(\mu)}
\end{aligned}
$$

and

$$
\widetilde{\hbar}_{22}(\mu)=\frac{1}{p^{-} \Delta^{-}(\mu)} \int_{a}^{c-} v^{-}(s, \mu) f(s) d s+\frac{f_{1}}{\Delta^{-}(\mu)}+\frac{f_{3}}{\gamma_{10}^{-}\left(\gamma_{21}^{-}-\mu^{2} \widetilde{\gamma}_{21}^{-}\right) \Delta^{-}(\mu)} .
$$

Putting in (26) gives

$$
u(x, \mu)=\left\{\begin{array}{rlrl}
\frac{\omega^{-}(x, \mu)}{p^{-} \Delta^{-}(\mu)} \int_{a}^{x} v^{-}(s, \mu) f(s) d s+\frac{v^{-}(x, \mu)}{p^{-} \Delta^{-}(\mu)} \int_{x}^{c-} \omega^{-}(s, \mu) f(s) d s & \\
& +v^{-}(x, \mu)\left(\frac{1}{p^{+} \Delta^{+}(\mu)} \int_{c^{+}}^{b} \omega^{+}(s, \mu) f(s) d s+\frac{f_{2}}{\Delta^{+}(\mu)}\right. & \\
& \left.+\frac{f_{3}}{\gamma_{10}^{+}\left(\gamma_{21}^{+}-\mu^{2} \widetilde{\gamma}_{21}^{+}\right) \Delta^{+}(\mu)}\right)+\omega^{-}(x, \mu) \frac{f_{1}}{\Delta^{-}(\mu)} & \\
\frac{\omega^{+}(x, \mu)}{p^{+} \Delta^{+}(\mu)} \int_{c+}^{x} v_{2}(s, \mu) f(s) d s+\frac{v^{+}(x, \mu)}{p^{+} \Delta^{+}(\mu)} \int_{x}^{b} \omega^{+}(s, \mu) f(s) d s & & \\
& +v^{+}(x, \mu) \frac{f_{2}}{\Delta^{+}(\mu)}+\omega^{+}(x, \mu)\left(\frac{1}{p^{-} \Delta^{-}(\mu)} \int_{a}^{c-} v^{-}(s, \mu) f(s) d s\right. & \\
& \left.\quad+\frac{f_{1}}{\Delta^{-}(\mu)}+\frac{f_{3}}{\gamma_{10}^{-}\left(\gamma_{21}^{-}-\mu^{2} \widetilde{\gamma}_{21}^{-} \Delta^{-}(\mu)\right.}\right) & \text { for } x \in \Omega^{+} .
\end{array}\right.
$$


Thus we find the needed resolvent function $u(x, \mu)$ in terms of the left- and right-define solutions $v^{ \pm}(x, \mu)$ and $\omega^{ \pm}(x, \mu)$. By introducing Green's function as

$$
G(x, s ; \mu)= \begin{cases}\frac{v^{-}(x, \mu) \omega^{-}(s, \mu)}{p^{-} \Delta^{-}(\mu)} & \text { if } x, s \in \Omega^{-}, s \geq x, \\ \frac{\omega^{-}(x, \mu) v^{-}(s, \mu)}{p^{-} \Delta-(\mu)} & \text { if } x, s \in \Omega^{-}, s<x, \\ \frac{v^{-}(x, \mu) \omega^{+}(s, \mu)}{p^{-}-\omega^{-}(\mu)} & \text { if } x \in \Omega^{-}, s \in \Omega^{+}, \\ \frac{\omega^{-}(x, \mu) v^{+}(s, \mu)}{p^{+} \Delta^{+}(\mu)} & \text { if } x \in \Omega^{+}, s \in \Omega^{-}, \\ \frac{\omega^{+}(x, \mu) v^{+}(s, \mu)}{p^{+} \Delta^{+}(\mu)} & \text { if } x \in \Omega^{+}, s \leq x, \\ \frac{v^{+}(x, \mu) \omega \omega^{+}(s, \mu)}{p^{+} \Delta^{+}(\mu)} & \text { if } x \in \Omega^{+}, s>x\end{cases}
$$

from (28) and (29) we have that the considered problem (21)-(22) has a unique solution given by

$$
\begin{aligned}
u(x, \mu)= & -\frac{1}{p^{-}}\left(\left|\theta_{34}\right|-\frac{\gamma_{10}^{-}}{\gamma_{10}^{+}}\left|\theta_{14}\right|\right) \int_{a}^{c-} G(x, s ; \mu) f(s) d s \\
& +\frac{1}{p^{+}}\left(\left|\theta_{12}\right|-\frac{\gamma_{10}^{+}}{\gamma_{10}^{-}}\left|\theta_{23}\right|\right) \int_{c+}^{b} G(x, s ; \mu) f(s) d s \\
& +\frac{1}{\rho_{1}}\left(\left|\theta_{34}\right|-\frac{\gamma_{10}^{-}}{\gamma_{10}^{+}}\left|\theta_{14}\right|\right) \widetilde{B}_{a}(G(x, \cdot ; \mu)) f_{1} \\
& -\frac{1}{\rho_{2}}\left(\left|\theta_{12}\right|-\frac{\gamma_{10}^{+}}{\gamma_{10}^{-}}\left|\theta_{23}\right|\right) \widetilde{B}_{b}(G(x, \cdot ; \mu)) f_{2} \\
& +\widetilde{T}_{c}(G(x, \cdot ; \mu)) f_{3} .
\end{aligned}
$$

\section{Representations of the resolvent operator in terms of Green's} vector-function

We now shall define Green's vector-function as follows:

$$
\widetilde{G}_{x, \mu}:=\left(G(x, \cdot ; \mu), \widetilde{B}_{a}(G(x, \cdot ; \mu)),-\widetilde{B}_{b}(G(x, \cdot ; \mu)), \widetilde{T}_{c}(G(x, \cdot ; \mu))\right) .
$$

Consequently, for the solution $U(F, \mu)$ of nonhomogeneous operator equation (21), we obtain the following formula:

$$
U(F, \mu)=\left(\left\langle\widetilde{G}_{x, \mu}, \bar{F}\right\rangle, \widetilde{B}_{a}\left(\left\langle\widetilde{G}_{x, \mu}, \bar{F}\right\rangle\right),-\widetilde{B}_{b}\left(\left\langle\widetilde{G}_{x, \mu}, \bar{F}\right\rangle\right), \widetilde{T}_{c}\left(\left\langle\widetilde{G}_{x, \mu}, \bar{F}\right\rangle\right)\right) .
$$

Using this, the resolvent function (30) can be written in the form

$$
u(x, \mu)=\left\langle\widetilde{G}_{x, \mu}, \bar{F}\right\rangle_{\mathcal{H}},
$$

where $\bar{F}=\left(\bar{f}(x), \bar{f}_{1}, \bar{f}_{2}, \bar{f}_{3}\right) \in \mathcal{H}$. Consequently, we have the following theorem.

Theorem 4 For the resolvent operator $R\left(\mu^{2}, \Re\right)=\left(\mu^{2} I-\Re\right)^{-1}$, the formula

$$
R\left(\mu^{2}, \Re\right) F=\left(\left\langle\widetilde{G}_{x, \mu}, \bar{F}\right\rangle, \widetilde{B}_{a}\left(\left\langle\widetilde{G}_{x, \mu}, \bar{F}\right\rangle\right),-\widetilde{B}_{b}\left(\left\langle\widetilde{G}_{x, \mu}, \bar{F}\right\rangle\right), \widetilde{T}_{c}\left(\left\langle\widetilde{G}_{x, \mu}, \bar{F}\right\rangle\right)\right),
$$

where $\bar{F}=\left(\bar{f}(x), \bar{f}_{1}, \bar{f}_{2}, \bar{f}_{3}\right) \in \mathcal{H}$, holds. 
Theorem 5 The estimation

$$
\left\|R\left(\mu^{2}, \Re\right) F\right\|_{\mathcal{H}} \leq\left|\operatorname{Im} \mu^{2}\right|^{-1}\|F\|_{\mathcal{H}}, \quad F \in \mathcal{H}
$$

holds for all regular value $\mu^{2}$ such that $\operatorname{Im} \mu^{2} \neq 0$.

Proof Let $F=\left(f(x), f_{1}, f_{2}, f_{3}\right) \in \mathcal{H}$. Denote $U=R\left(\mu^{2}, \Re\right) F$. Since $\Re U=\mu^{2} U-F$, taking into account that the operator $\Re$ is symmetric, we have

$$
\mu^{2}\langle U, U\rangle_{\mathcal{H}}-\langle F, U\rangle_{\mathcal{H}}=\langle\Re U, U\rangle_{\mathcal{H}}=\langle U, \Re U\rangle_{\mathcal{H}}=\bar{\mu}^{2}\langle U, U\rangle_{\mathcal{H}}-\overline{\langle F, U\rangle_{\mathcal{H}}}
$$

Using the well-known Cauchy-Schwarz inequality, we conclude that

$$
\left|\operatorname{Im} \mu^{2}\right|\|U\|_{\mathcal{H}}^{2}=|\operatorname{Im}\langle F, U\rangle|_{\mathcal{H}} \leq\|F\|_{\mathcal{H}}\|U\|_{\mathcal{H}} .
$$

Consequently,

$$
\left\|R\left(\mu^{2}, \Re\right) F\right\|_{\mathcal{H}} \leq\left|\operatorname{Im} \mu^{2}\right|^{-1}\|F\|_{\mathcal{H}} .
$$

The proof is complete.

Theorem 6 The resolvent operator $R\left(\mu^{2}, \Re\right)$ is compact in the Hilbert space $\mathcal{H}$.

Proof Let $\lambda_{1} \leq \lambda_{2} \leq \cdots$ be eigenvalues of $\Re$ and let $E_{\lambda_{1}}, E_{\lambda_{2}}, \ldots$ be orthogonal projections onto the corresponding eigenspace. Since $\Re$ is a self-adjoint operator with discrete spectrum, we can write the spectral resolution of the resolvent operator $R\left(\mu^{2}, \Re\right)$ by

$$
R\left(\mu^{2}, \Re\right)=\sum_{n=1}^{\infty}\left(\lambda_{n}-\mu^{2}\right)^{-1} E_{\lambda_{n}}
$$

Similarly to [22] we can easily show that $\lambda_{n}=O\left(n^{2}\right)$. Thus $\left(\lambda_{n}-\mu^{2}\right)^{-1}=O\left(n^{-2}\right)$ for $n \rightarrow \infty$. Consequently, the series (35) is strongly convergent. It is obvious that the orthogonal projections $E_{\lambda_{n}}, n=1,2, \ldots$, are compact operators since each of them are of finite rank. Consequently, the sum of series (35) is also compact in $\mathcal{H}$. The proof is complete.

\section{Competing interests}

The author declares that she has no competing interests.

\section{Acknowledgements}

The author is grateful to anonymous referees for their constructive comments and suggestions, which led to the improvement of the original manuscript.

Received: 4 March 2014 Accepted: 8 May 2014 Published: 22 May 2014

\section{References}

1. Levitan, BM, Sargsyan, IS: Sturm-Liouville and Dirac Operators. Springer, New York (1991)

2. Pryce, JD: Numerical Solution of Sturm-Liouville Problems. Monographs on Numerical Analysis. Oxford Science Publications. Oxford University Press, New York (1993)

3. Hinton, D, Schaefer, PW: Spectral Theory and Computational Methods for Sturm-Liouville Problems. Lect. Notes Pure Appl. Math., vol. 191. Dekker, New York (1997)

4. Titchmarsh, EC: Eigenfunction Expansions Associated with Second-Order Differential Equations. Part I, 2nd edn. Oxford University Press, London (1962) 
5. Zettl, A: Sturm-Liouville Theory. Mathematical Surveys and Monographs, vol. 121. Am. Math. Soc., Providence (2005)

6. Duffy, DG: Green's Functions with Applications. Chapman \& Hall/CRC, Boca Raton (2001)

7. Stakgold, I, Holst, MJ: Green's Functions and Boundary Value Problems, 3rd edn. Wiley, New York (2011)

8. Ao, J, Sun, J, Zhang, M: Matrix representations of Sturm-Liouville problems with transmission conditions. Comput. Math. Appl. 63, 1335-1348 (2012)

9. Aydemir, K, Mukhtarov, OS: Green's function method for self-adjoint realization of boundary-value problems with interior singularities. Abstr. Appl. Anal. 2013, Article ID 503267 (2013). doi:10.1155/2013/503267

10. Kong, Q, Wang, Q: Using time scales to study multi-interval Sturm-Liouville problems with interface conditions. Results Math. 63, 451-465 (2013)

11. Mukhtarov, OS, Aydemir, K: New type Sturm-Liouville problems in associated Hilbert spaces. J. Funct. Spaces Appl. 2014, Article ID 606815 (2014)

12. Voitovich, NN, Katsenelbaum, BZ, Sivov, AN: Generalized Method of Eigen-Vibration in the Theory of Diffraction. Nauka, Moscow (1997) (Russian)

13. Likov, AV, Mikhailov, YA: The Theory of Heat and Mass Transfer. Qosenergaizdat, Moscow (1963) (Russian)

14. Tikhonov, AN, Samarskii, AA: Equations of Mathematical Physics. Pergamon, New York (1963)

15. Albeverio, S, Gesztesy, F, Hoegh Krohn, R, Holden, H: Solvable Models in Quantum Mechanics, 2nd edn. AMS Chelsea Publishing, Providence (2005)

16. Titeux, I, Yakubov, Y: Completeness of root functions for thermal conduction in a strip with piecewise continuous coefficients. Math. Models Methods Appl. Sci. 7, 1035-1050 (1997)

17. Mukhtarov, OS, Demir, H: Coerciveness of the discontinuous initial-boundary value problem for parabolic equations. Isr. J. Math. 114, 239-252 (1999)

18. Mukhtarov, OS, Yakubov, S: Problems for ordinary differential equations with transmission conditions. Appl. Anal. 81 1033-1064 (2002)

19. Rasulov, ML: Methods of Contour Integration. North-Holland, Amsterdam (1967)

20. Akdoğan, Z, Demirci, M, Mukhtarov, OS: Green function of discontinuous boundary-value problem with transmission conditions. Math. Methods Appl. Sci. 30, 1719-1738 (2007)

21. Bairamov, $\mathrm{E}:$ On the characteristic values of the real component of a dissipative boundary value transmission problem. Appl. Math. Comput. 218, 9657-9663 (2012)

22. Mukhtarov, OS, Kadakal, M: Some spectral properties of one Sturm-Liouville type problem with discontinuous weight. Sib. Math. J. 46, 681-694 (2005)

23. Altınışık, N, Mukhtarov, OS, Kadakal, M: Asymptotic formulas for eigenfunctions of the Sturm-Liouville problems with eigenvalue parameter in the boundary conditions. Kuwait J. Sci. Eng. 39, 1-19 (2012)

24. Muhtarov, FS, Aydemir, K: Distributions of eigenvalues for Sturm-Liouville problem under jump conditions. J. New Results Sci. 1, 81-89 (2012)

25. Uğurlu, E, Bairamov, E: Dissipative operators with impulsive conditions. J. Math. Chem. 51, 1670-1680 (2013)

10.1186/1687-2770-2014-131

Cite this article as: Aydemir: Boundary value problems with eigenvalue-dependent boundary and transmission conditions. Boundary Value Problems 2014, 2014:131

\section{Submit your manuscript to a SpringerOpen ${ }^{\circ}$ journal and benefit from:}

- Convenient online submission

- Rigorous peer review

- Immediate publication on acceptance

- Open access: articles freely available online

- High visibility within the field

- Retaining the copyright to your article 